\title{
A Review of Cost-Effectiveness Studies of Pembrolizumab Regimens for the Treatment of Advanced Non-small Cell Lung Cancer
}

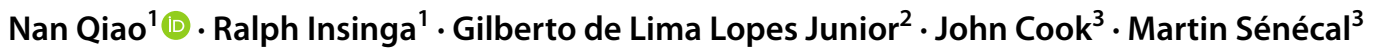

Accepted: 27 December 2020 / Published online: 19 January 2021

(c) The Author(s) 2021

\begin{abstract}
Pembrolizumab monotherapy or combination therapy is an approved treatment for various advanced non-small cell lung cancer (NSCLC) indications. We review published cost-effectiveness analyses (CEAs) of pembrolizumab as treatment for NSCLC and provide in-depth assessment of their methodologies. Fourteen studies were selected through searches of the PubMed database. Modeling approaches, survival and cost estimation, and utility analyses were compared and evaluated. These publications covered regulatory-approved pembrolizumab NSCLC indications based on the following randomized clinical trials: KEYNOTE-010 (one publication), KEYNOTE-024 (six), KEYNOTE-042 (four), KEYNOTE-189 (two), and KEYNOTE-407 (one). Differences were observed in health states (progression free, progressed disease, and death vs stable disease, progressed disease, death, and treatment discontinuation), modeling approaches (partitioned survival vs Markov), survival extrapolation/transition probability estimation, inclusion of additional costs to drug, disease management and adverse event costs (e.g., programmed death-ligand 1 [PD-L1] testing, subsequent treatment, terminal care), treatment duration approaches (trial-based time on treatment vs treat to progression), utility sources (trial data vs literature), and utility analyses (time to death vs progression status). Certain aspects of variability across models were problematic, including deviation from observed treatment utilization within trials and predicted long-term mortality risks for pembrolizumab higher than historical real-world NSCLC mortality data prior to the availability of pembrolizumab. Consequently, results differed even among studies examining the same population and comparator within similar time intervals. Differences in methodology across CEAs may lead to distinct results and conclusions. Payers and policy makers should carefully examine study designs and assumptions and choose CEAs with greater validity and accuracy for evidence-based decision-making.
\end{abstract}

Nan Qiao

nan.qiao@merck.com

Ralph Insinga

ralph_insinga@merck.com

Gilberto de Lima Lopes Junior

glopes@med.miami.edu

John Cook

john.cook@cheors.com

Martin Sénécal

martin.senecal@cheors.com

1 Center for Observational and Real-World Evidence, Merck \& Co., Inc., Kenilworth, NJ 07033, USA

2 Clinical Oncology Sector, University of Miami, 1120 NW 14th St, Suite 650J, Miami, FL 33136, USA

3 Complete HEOR Solutions, 1120 Welsh Rd \#205, North Wales, PA 19454, USA 


\section{Key Points for Decision Makers}

The detailed methodologies and results of 14 cost-effectiveness analysis (CEA) publications of pembrolizumab trials in non-small cell lung cancer (NSCLC) were reviewed and compared.

Differences in methodology can potentially lead to opposing conclusions on the cost-effectiveness of NSCLC therapies.

Policy makers must weigh the limitations of CEA designs to make informed decisions.

\section{Introduction}

Lung cancer is the most common cancer type and cause of cancer death worldwide [1]. Non-small cell lung cancer (NSCLC) accounts for $80-85 \%$ of all lung cancers and can be further divided into squamous and nonsquamous subtypes. NSCLC often does not show symptoms until advanced stages [2]. Nearly $70 \%$ of diagnosed cases are locally advanced or metastatic [3].

Historically, late-stage NSCLC was treated with chemotherapy, which led to an overall survival (OS) of 8-10 months [4]. Immune checkpoint inhibitors are a breakthrough cancer treatment, significantly improving OS and/ or progression-free survival (PFS) over chemotherapy for patients with advanced NSCLC in multiple clinical trials [5].

Pembrolizumab, an anti-programmed death-1 (anti-PD-1) monoclonal antibody, was initially approved as a monotherapy by the United States (US) Food and Drug Administration (FDA) in the second- and later-line settings for patients with metastatic NSCLC based on programmed death-ligand 1 (PD-L1) tumor proportion score (TPS) (TPS $\geq 50 \%$ based on KEYNOTE-001, and TPS $\geq 1 \%$ based on KEYNOTE-010) [6, 7]. Subsequently, first-line pembrolizumab monotherapy was approved for patients with metastatic NSCLC with a TPS of $\geq 50 \%$ and no epidermal growth factor receptor or anaplastic large-cell lymphoma kinase (EGFR-/ALK-) genomic tumor aberrations (KEYNOTE-024) [7]. Today, pembrolizumab monotherapy is a first-line standard of care for patients with metastatic NSCLC with a TPS of $\geq 1 \%$ and patients with locally advanced NSCLC with a TPS of $\geq 1 \%$ who are ineligible for surgical resection or definitive chemoradiation (KEYNOTE-042) [8]. First-line pembrolizumab plus chemotherapy combinations are a standard of care for $E G F R-/ A L K-$ nonsquamous metastatic NSCLC (KEYNOTE-189) and squamous metastatic NSCLC (KEYNOTE-407) [8]. Regulatory agencies in many other countries have also approved pembrolizumab with or without chemotherapy as a treatment for all or a portion of these FDA-approved NSCLC indications.

The economic value of pembrolizumab-based regimens versus other treatments for NSCLC has been examined in multiple cost-effectiveness analyses (CEAs). These analyses differed in modeling approaches, survival and cost estimation, and/or utility analyses, yielding varied results and conclusions even for identical patient populations and treatments. Healthcare payers rely on CEA results to make coverage and reimbursement decisions. Here, we present a review that examines CEA methodologies in depth and discusses how they may affect study findings.

As of this writing, two literature reviews of the economic value of NSCLC treatment with immunotherapy have been published $[9,10]$. da Veiga et al. conducted a meta-narrative review on the costs and economic value of pembrolizumab and nivolumab in treating melanoma, NSCLC, and renal cell carcinoma, as well as using PD-L1 testing to select NSCLC patients eligible for immunotherapy. They found contradictory results from three published CEAs studying nivolumab as treatment for advanced NSCLC in Saudi Arabia, Canada, and the US, and attributed differences to the choice of chemotherapy comparator [9]. Verma et al. systematically reviewed published costs and CEAs of immunotherapies such as pembrolizumab for treatment of head and neck cancers, NSCLC, genitourinary cancers, and melanoma, as well as using PD-L1 testing to identify eligible patients. They listed results from previously published CEAs comparing immuno- and chemotherapy treatment for NSCLC and concluded that nivolumab was only cost-effective above certain PD-L1 levels, while pembrolizumab was cost-effective for both previously treated and treatment-naïve patients with NSCLC [10]. Neither review examined modeling methodologies before reaching their conclusions.

\section{Materials and Methods}

This study aims to compare methodologies and findings in model-based CEAs of pembrolizumab with/without chemotherapy for treating advanced NSCLC. The eligibility criteria for a publication to be included are as follows:

- Study population included patients with advanced/metastatic NSCLC.

- Interventions included pembrolizumab regimen(s).

- Study type was CEA.

- Study designs included modeling and simulation. 
- Outcomes included incremental cost-effectiveness ratios (ICERs).

- Published in the English language.

A PubMed search was performed using the following strategy: (pembrolizumab) AND (non-small-cell lung carcinoma OR non-small cell lung carcinoma OR non-smallcell lung cancer OR non-small cell lung cancer OR NSCLC) AND (cost-effectiveness OR cost effectiveness) with limits to English-language publications that were published through to December 10, 2019. The search yielded 21 studies. One author examined titles/abstracts/full texts to determine eligibility and excluded seven studies. The excluded studies and the reasons for exclusion were as follows: da Veiga et al. and Verma et al. were CEA literature reviews [9, 10]; Bravaccini, Norum et al. and Tartari et al. were not CEAs [11-13]; and Aguiar et al. did not include modeling and simulation [14, 15]. The remaining 14 studies were reviewed in detail. One author extracted data, and a second author cross-checked the extracted information for accuracy. Different findings between the two authors were resolved via consultation with other authors.

Figure 1 outlines the selection process of the included publications.

\section{Results}

\subsection{Overview}

Table 1 provides an overview of the 14 publications and shows base-case results. These studies covered regulatoryapproved pembrolizumab NSCLC indications from a wide geographic area. The most commonly used perspectives were payer (eight of 14 studies) and healthcare system (six of 14 studies), within which Georgieva et al. took both a UK payer and a US healthcare system perspective [16]. The major difference between payer and healthcare system perspectives is that the former includes medical costs paid by payers only, while the latter includes medical costs paid by payers or patients [17]. Two studies from a US or Chinese payer perspective did not report the application of coinsurance rates to cost calculations (Table 1) [18, 19].

The base-case time horizon applied in these studies varied from 10 years to a lifetime. Some studies explicitly listed the evidence used to support their time horizon choice: Huang et al. consistently applied a 20 -year time horizon in the base case, as the extrapolated OS projected that only $0.7 \%$ of KEYNOTE-010 pembrolizumab-treated patients were still alive 20 years after treatment onset [20]. Insinga et al. extrapolated OS curves for the pembrolizumab combination arm in the KEYNOTE-189 and KEYNOTE-407 trial populations. Based on their models, approximately $10 \%$ and $<5 \%$ of patients remained alive 10 and 20 years after initiating pembrolizumab plus chemotherapy, respectively, and thus 20 years was chosen

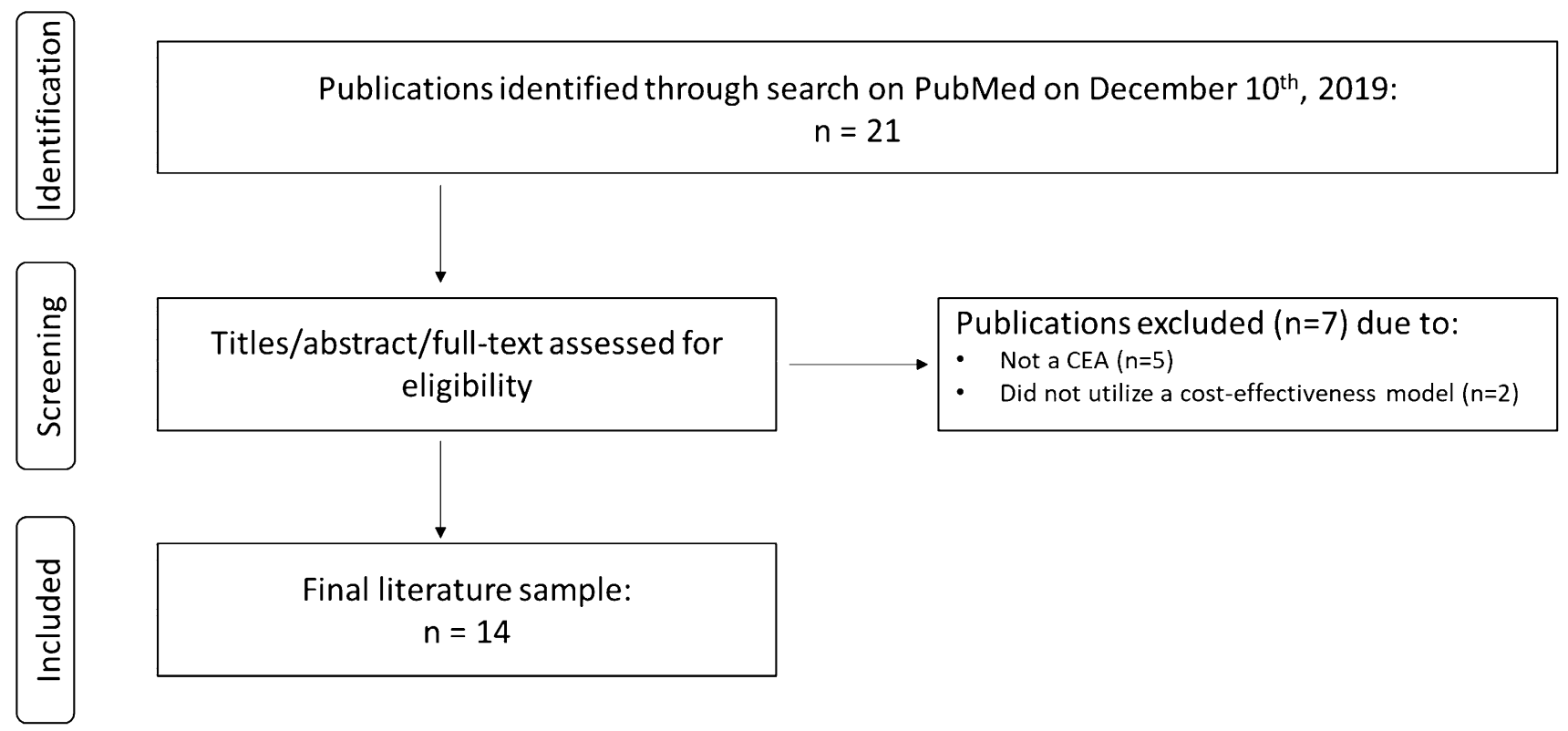

Fig. 1 Literature selection flow chart. $C E A$ cost-effectiveness analysis 
Table 1 Summary of included cost-effectiveness analyses

\begin{tabular}{|c|c|c|c|c|c|c|c|}
\hline References & Population $^{\mathrm{a}}$ & Perspective & Treatment line & Intervention & $\begin{array}{l}\text { Comparator(s) } \\
\text { (main comparator } \\
\text { listed first) }\end{array}$ & $\begin{array}{l}\text { Time horizon } \\
\text { (base case) }\end{array}$ & $\begin{array}{l}\text { ICER(s) vs main } \\
\text { comparator }\end{array}$ \\
\hline Chouaid et al. [28] & KN024 & $\begin{array}{l}\text { French health } \\
\text { system }\end{array}$ & $1 \mathrm{~L}$ & Pembro mono & $\begin{array}{l}\text { Trial chemo, } \\
\text { NMA with } \\
\text { beva-containing } \\
\text { regimens in } \\
\text { NSQ patients }\end{array}$ & 10 years & $\begin{array}{l}€ 84 \mathrm{~K} / \mathrm{QALY} \text { (SQ) } \\
€ 79 \mathrm{~K} / \mathrm{QALY}(\mathrm{NSQ})\end{array}$ \\
\hline Criss et al. [31] & $\begin{array}{l}\text { KN189/ } \\
\text { IMpower150 }\end{array}$ & $\begin{array}{l}\text { US healthcare } \\
\text { sector }\end{array}$ & $1 \mathrm{~L}$ & Pembro combo & $\begin{array}{l}\text { Atezo combo, } \\
\text { KN189/ } \\
\text { IMpower150 } \\
\text { chemo }\end{array}$ & NR & Dominant \\
\hline $\begin{array}{l}\text { Georgieva et al. } \\
\text { [16] }\end{array}$ & KN024 & $\begin{array}{l}\text { UK NHS and US } \\
\text { cost }\end{array}$ & $1 \mathrm{~L}$ & Pembro mono & Trial chemo & $\begin{array}{l}\text { Until death or } \\
\text { treatment dis- } \\
\text { continuation }\end{array}$ & $\begin{array}{l}\text { \$52k/QALY (UK) } \\
\text { \$49k/QALY (US) }\end{array}$ \\
\hline $\mathrm{Hu}$ and Hay [23] & KN024 & UK healthcare & $1 \mathrm{~L}$ & Pembro mono & Trial chemo & $\begin{array}{l}\text { Until } 99 \% \text { of } \\
\text { patients die }\end{array}$ & $£ 87 \mathrm{~K} / \mathrm{QALY}$ \\
\hline Huang et al. [20] & KN010 & $\begin{array}{r}\text { US payer }(20 \% \\
\text { coinsurance) }\end{array}$ & $2 \mathrm{~L}$ & Pembro mono & Trial chemo & 20 years & \$169K/QALY \\
\hline Huang et al. [24] & KN024 & $\begin{array}{l}\text { US public payer } \\
\text { ( } 20 \% \text { coinsur- } \\
\text { ance) }\end{array}$ & $1 \mathrm{~L}$ & Pembro mono & Trial chemo & 20 years & \$98K/QALY \\
\hline Huang et al. [26] & KN042 & $\begin{array}{l}\text { US public payer } \\
\text { ( } 20 \% \text { coinsur- } \\
\text { ance) }\end{array}$ & $1 \mathrm{~L}$ & Pembro mono & Trial chemo & 20 years & $\begin{array}{l}\$ 130 \mathrm{~K} / \mathrm{QALY} \text { (TPS } \\
\geq 1 \% \text { ) } \\
\$ 112 \mathrm{~K} / \mathrm{QALY} \text { (TPS } \\
\geq 50 \%)\end{array}$ \\
\hline Insinga et al. [21] & KN189 & $\begin{array}{l}\text { US payer }(20 \% \\
\text { coinsurance) }\end{array}$ & $1 \mathrm{~L}$ & Pembro combo & $\begin{array}{l}\text { Trial chemo, } \\
\text { pembro mono } \\
\text { for TPS } \geq 50 \%\end{array}$ & 20 years & \$105K/QALY \\
\hline Insinga et al. [22] & KN407 & $\begin{array}{r}\text { US payer ( } 20 \% \\
\text { coinsurance) }\end{array}$ & $1 \mathrm{~L}$ & Pembro combo & $\begin{array}{l}\text { Trial chemo, } \\
\text { pembro mono } \\
\text { for TPS } \geq 50 \%\end{array}$ & 20 years & \$86K/QALY \\
\hline Liao et al. [30] & KN024 & Chinese society & $1 \mathrm{~L}$ & Pembro mono & Trial chemo & 10 years & \$103K/QALY \\
\hline Loong et al. [29] & KN024 & $\begin{array}{l}\text { Hong Kong hospi- } \\
\text { tal authority }\end{array}$ & $1 \mathrm{~L}$ & $\begin{array}{l}\text { BTS: TPS } \geq 50 \% \\
\text { receiving } \\
\text { pembro and trial } \\
\text { chemo otherwise }\end{array}$ & $\begin{array}{l}\text { All patients } \\
\text { receiving trial } \\
\text { chemo }\end{array}$ & 10 years & HK\$865K/QALY \\
\hline She et al. [18] & KN042 & US payer & $1 \mathrm{~L}$ & Pembro mono & Trial chemo & 20 years & $\begin{array}{l}\$ 136 \mathrm{~K} / \mathrm{QALY} \text { (TPS } \\
\geq 50 \% \text { ) } \\
\$ 161 \mathrm{~K} / \mathrm{Q} A L Y \text { (TPS } \\
\geq 20 \% \text { ) } 180 \mathrm{~K} / \\
\text { QALY (TPS } \geq \\
1 \% \text { ) }\end{array}$ \\
\hline Weng et al. [25] & KN042 & $\begin{array}{l}\text { US healthcare } \\
\text { system }\end{array}$ & $1 \mathrm{~L}$ & Pembro mono & Trial chemo & $\begin{array}{l}\text { Until } 99 \% \text { of } \\
\text { patients die }\end{array}$ & $\begin{array}{l}\$ 48 \mathrm{~K} / \mathrm{QALY} \text { (TPS } \\
\geq 50 \% \text { ) } \\
\$ 47 \mathrm{~K} / \mathrm{QALY} \text { (TPS } \\
\geq 20 \% \text { ) } \\
\text { \$68K/QALY (TPS } \\
\geq 1 \% \text { ) }\end{array}$ \\
\hline Zhou et al. [19] & KN042 & Chinese payer & $1 \mathrm{~L}$ & Pembro mono & Trial chemo & 10 years & $\begin{array}{l}\$ 36 \mathrm{~K} / \mathrm{QALY} \text { (TPS } \\
\geq 50 \% \text { ) } \\
\$ 42 \mathrm{~K} / \mathrm{QALY} \text { (TPS } \\
\geq 20 \% \text { ) } \\
\text { \$39K/QALY (TPS } \\
\geq 1 \% \text { ) }\end{array}$ \\
\hline
\end{tabular}

$1 L$ first line, $2 L$ second line, $A L K$ anaplastic lymphoma kinase, atezo atezolizumab, beva bevacizumab, BTS biomarker (PD-L1) test-and-treat strategy, chemo chemotherapy, combo combination, EGFR epidermal growth factor receptor, $H K$ Hong Kong, ICER incremental cost-effectiveness ratio, KN KEYNOTE, mono monotherapy, NHS National Health Service, NMA network meta-analysis, $N R$ not reported, $N S C L C$ non-small cell lung cancer, $N S Q$ nonsquamous, $P D-L 1$ programmed death-ligand 1, pembro pembrolizumab, $Q A L Y$ quality-adjusted life-year, $S Q$ squamous, TKI tyrosine kinase inhibitor, TPS tumor proportion score, UK United Kingdom, US United States

${ }^{a}$ Trial study populations: IMpower150: metastatic NSQ NSCLC; KN010: $E G F R-/ A L K-$ advanced NSCLC progressing after platinum therapy or EGFR/ALK+ advanced NSCLC progressing after TKI treatment; KN024: EGFR-/ALK- metastatic NSCLC with a TPS of $\geq 50 \%$; KN042: $E G F R-/ A L K-$ metastatic NSCLC or advanced NSCLC not eligible for surgery/definitive chemoradiation with a TPS of $\geq 1 \%$; KN189: EGFR-/ $A L K-$ metastatic NSQ NSCLC; KN407: metastatic SQ NSCLC 
as the base case and 10 years used as a scenario. Estimated ICERs were decreased from $\$ 119 \mathrm{~K} /$ quality-adjusted lifeyear (QALY) to \$105K/QALY and from \$103K/QALY to $\$ 86 \mathrm{~K} / \mathrm{QALY}$ at 10 and 20 years for KEYNOTE-189 and KEYNOTE-407 patients, respectively [21, 22]. Based on these comparisons, a 10-year time horizon may not fully capture lifetime costs and health benefits of pembrolizumab-based therapy and a $\geq 20$-year time horizon is warranted. CEA publications should report proportions of surviving patients in all arms when fixed time horizons are used.

Results could vary even across CEAs of the same trial population. For example, among CEAs based on KEYNOTE-024, ICERs varied from £43K/QALY (lifetime horizon) to $£ 87 \mathrm{~K} / \mathrm{QALY}$ (until $99 \%$ of patients died) for the UK population and from \$49K/QALY (lifetime) to \$98K/QALY (20 years) for the US population [16, 23, 24]. Among three US-based KEYNOTE-042 CEAs, ICERs varied from \$48K/ QALY (until 99\% of patients died) to \$136K/QALY (20 years) for patients with a TPS of $\geq 50 \%$, from $\$ 47 \mathrm{~K} / \mathrm{QALY}$ (until 99\% of patients died) to $\$ 161 \mathrm{~K} / \mathrm{QALY}$ (20 years) for patients with a TPS of $\geq 20 \%$, and from \$68K/QALY (until $99 \%$ of patients died) to $\$ 180 \mathrm{~K} / \mathrm{QALY}$ (20 years) for patients with a TPS of $\geq 1 \%[18,25,26]$.

According to the International Society for Pharmacoeconomics and Outcomes Research (ISPOR)-Society for Medical Decision Making (SMDM) Modeling Good Research Practices Task Force, examining and reporting uncertainty is a very important aspect of cost-effectiveness modeling [27]. The most commonly conducted uncertainty analyses in cost-effectiveness modeling are deterministic sensitivity analysis (DSA) that assesses uncertainty related to one or a set of parameters with continuous values, probabilistic sensitivity analysis (PSA) that assesses uncertainty by varying all continuous variables simultaneously using a simulation, and scenario analyses that assess uncertainty related to parameters with discrete values [27]. Among the 14 studies, eight performed one-way DSA, PSA, and scenario analyses [20-24, 26, 28, 29]. Another five studies performed DSA and PSA, but not scenario analyses [18, 19, 25, 30, 31]. Georgieva et al. performed a sensitivity analysis on prior distribution of survival and study-to-study heterogeneity and also conducted scenario analyses on discount rates and distribution for the survival model for their Bayesian Markov model [16].

Among the 13 studies that conducted DSA, 11 used 95\% confidence intervals for extreme value tests when available [20-22, 24-26, 28, 29, 31], while Hu and Hay and Zhou et al. only made assumptions on the variation range of the tested parameters, which were $\pm 20 \%$ and $\pm 30 \%$, respectively [19, 23]. Nine CEAs specified parametric distribution use in the PSA [18, 20-22, 24-26, 29, 30].

\subsection{Modeling Approaches and Survival Extrapolations}

Table 2 summarizes the modeling approaches and survival extrapolation methods used in the 14 studies.

\subsubsection{Modeling Approaches}

Seven studies applied a partitioned-survival modeling approach, while the other seven applied one of several Markov-related approaches (two Markov, three semiMarkov, one patient-level state transition, and one Bayesian Markov).

All approaches assumed disease progression is irreversible, i.e., patients cannot move from progressed disease (PD) to progression free (PF). While partitioned-survival models incorporated time dependency in extrapolated PFS and OS curves, the three semi-Markov, one patient-level simulation, and the Bayesian Markov models also used survival functions to estimate transition probabilities over time to incorporate time dependency [16, 18, 23, 25, 31]. In contrast, Liao et al. and Zhou et al. assumed constant transition probabilities over time in their Markov models [19, 30].

Partitioned-survival models use PFS and OS KaplanMeier (KM) data from clinical trials and therefore can model survival functions precisely during the trial period, which is more difficult to achieve within a Markov modeling framework. On the other hand, Markov models make patients' transitions explicit, while partitioned-survival models only provide a Markov trace but not a transition matrix. Thus, while both models report the number of deaths to occur in a given cycle, the transition matrix allows one to determine how many of these deaths were among individuals previously in the PF or PD health state.

Partitioned-survival and Markov models applied in some of the studies assumed structurally unrelated survival functions for each treatment arm, and therefore survival parameters cannot easily be varied dependently in PSAs. Some partitioned-survival models applied a relative risk of mortality between treatment arms. For example, three studies applied the same constant hazard within both arms, estimated from the Surveillance Epidemiology and End Results (SEER) program, when extrapolating OS beyond the trial period $[20,24,26]$. In contrast, two studies applied SEER-based mortality risks in the chemotherapy arm for long-term OS prediction and then an efficacy relative risk to the chemotherapy arm mortality risks to derive long-term OS predictions for the pembrolizumab plus chemotherapy arm, thus allowing dependence of OS between comparator arms [21, 22]. Among the Markov models, Criss et al. also applied SEER-based mortality risks across arms after year 5 when extrapolating OS curves [31]. Georgieva et al. allowed 


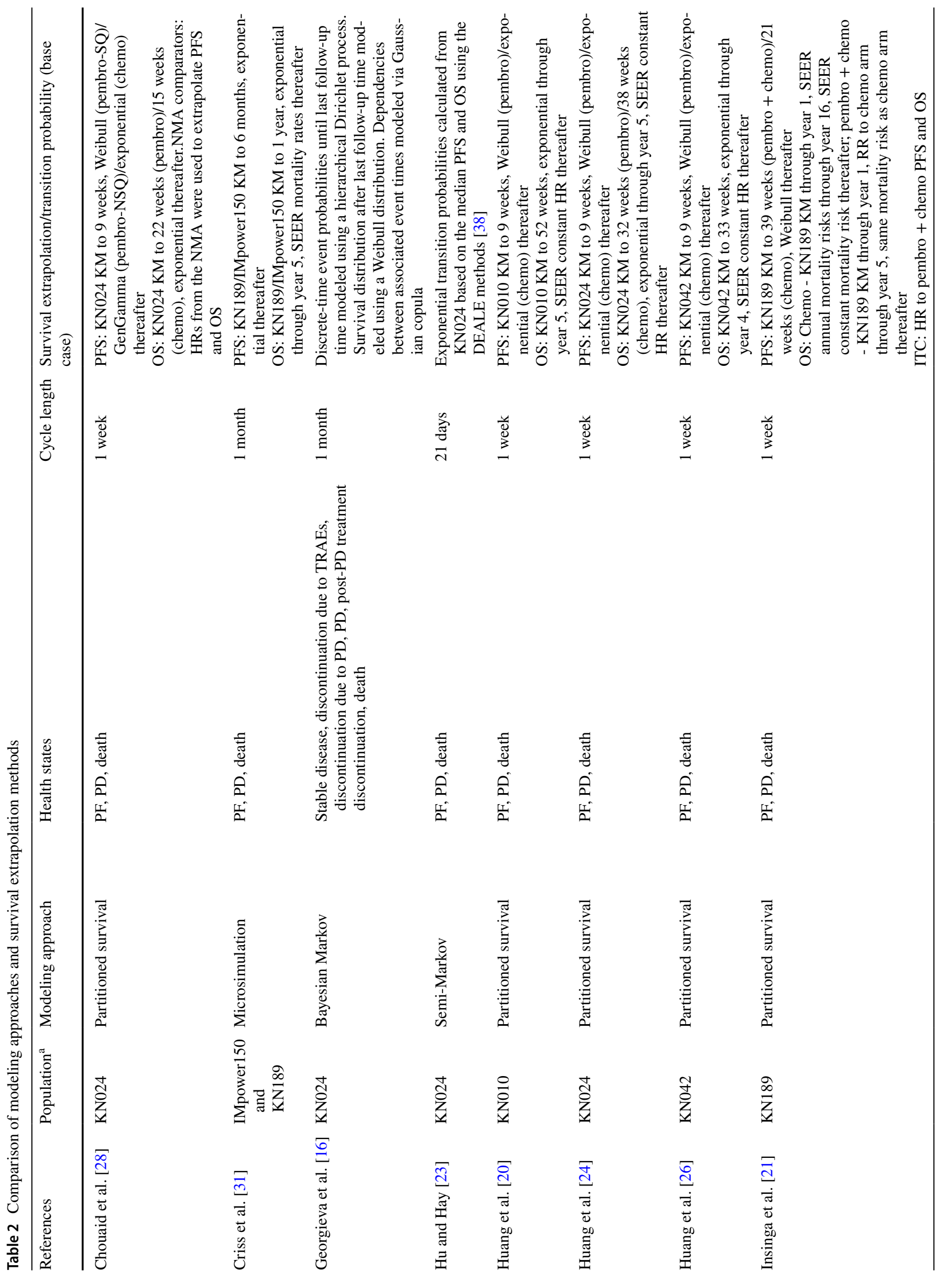




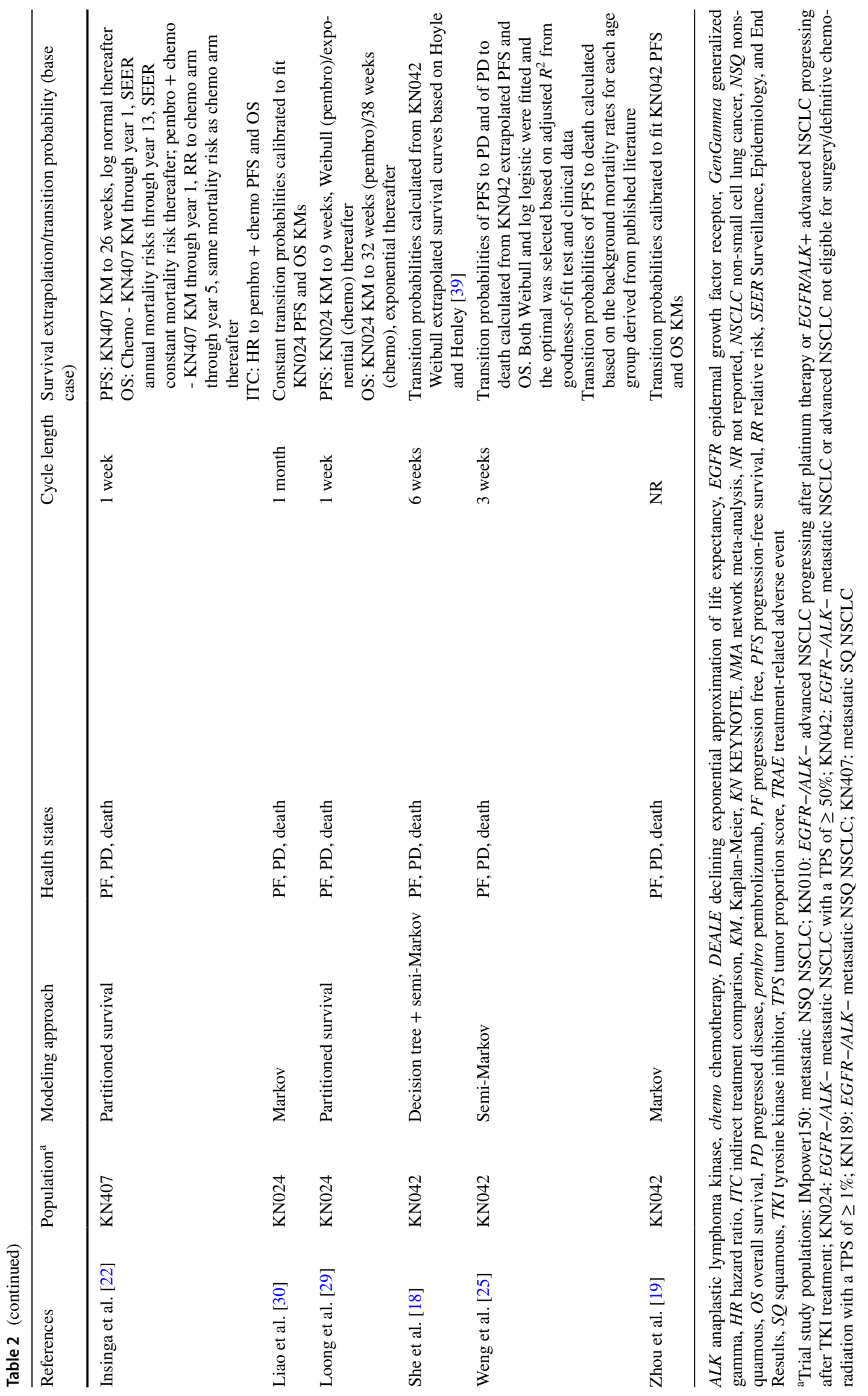


dependency between arms and between progression and OS by using a Gaussian copula in their Bayesian Markov model [16].

Each modeling approach has strengths and weaknesses. Researchers should select the approach that best fits their study purposes while taking its limitations into consideration. Time dependency of survival should reflect the realworld disease progression process. Survival parameters should have the option to vary dependently across treatment arms, as third factors such as trial inclusion/exclusion criteria may affect survival of both arms.

\subsubsection{Health States}

Most studies included three health states commonly applied in oncology models, i.e., PF, PD, and death. Georgieva et al. included stable disease, PD, and death, but also added discontinuation due to treatment-related adverse events (TRAEs), discontinuation due to progression, and postdiscontinuation treatment discontinuation as three alternative absorbing states other than death [16]. Based on KEYNOTE-024 trial results, Georgieva et al. assigned 14\% of pembrolizumab-treated patients and $11 \%$ of chemotherapytreated patients to discontinue treatment due to TRAEs, and $56 \%$ of pembrolizumab-treated patients and $46 \%$ of chemotherapy-treated patients to discontinue treatment upon progression. After disease progression, pembrolizumabtreated and chemotherapy-treated patients were assumed to discontinue treatment after a median of four and five cycles, respectively [16]. As a higher proportion of patients in the pembrolizumab arm entered into the three treatment discontinuation absorbing states than patients in the chemotherapy arm and were not allowed to transition to the death state afterwards, their survival was overestimated. Setting the three treatment discontinuation states as non-absorbing states and allowing patients to transition to death can reduce bias.

\subsubsection{Cycle Length}

Cycle lengths varied across analyses, with intervals of 1 week, 3 weeks, 1 month, or 6 weeks. Without affecting computational efficiency, a shorter cycle length is preferred to provide more flexibility in capturing the actual interval of treatment, which can differ among comparators and increase estimation precision.

\subsubsection{Estimation of PFS/OS}

In the studies utilizing partitioned survival models, different assumptions were made related to survival prediction beyond the trial observation period. All studies used a piecewise model to extrapolate survival curves so that the original PFS and OS KM curves could be used within the trial period. As the best fitting parametric approach predicted higher annual mortality risks in the medium- to long-term than mortality observed from SEER data, some studies used SEER data in longer-term OS prediction to avoid overestimating NSCLC mortality [20-22, 24, 26]. For the Markov models, the probabilities were based on the PFS and OS trial survival curves, their exponential or Weibull extrapolation, or extrapolation based on a piecewise model encompassing long-term SEER mortality data. Regardless of the applied survival prediction method, it is difficult to assess the plausibility of predicted survival curves beyond the trial period without long-term trial or observational data.

An overreliance on statistical fitting criteria for generating extrapolated survival curves has been observed in some studies. Parametric statistical fitting can suggest a statistical distribution that fits a limited short-term observation window and yields a survival curve that reliably matches trial KMs, but clinical considerations should govern choices made for longer-term survival extrapolation. The exponential distribution was selected for OS extrapolation in five partitioned-survival analyses and one patient-level simulation analysis mainly based on statistical fitting criteria and visual inspection [20, 24, 26, 28, 29, 31]. However, the exponential distribution assumes a constant risk of death across time, while NSCLC mortality risks are observed to decline over time in population-based data, likely due to surviving patients increasingly reflecting those with long-term remission or cure (complete remission $\geq 5$ years) or who otherwise are in better general health (hardy survivor population) $[21,22]$. Therefore, applying the exponential distribution for extrapolation is likely to underestimate the long-term survival potential and cost-effectiveness of pembrolizumabbased therapies.

Extrapolated annual mortality risks in the trial control arm should not exceed those observed in historical population-based data for metastatic NSCLC patients, such as SEER. Six studies chose to use population-based NSCLC mortality risks directly in long-term modeling [20-22, 24, $26,31]$, while the rest relied on parametric statistical fitting and seldom compared the extrapolated mortality risks with those observed in population data [16, 18, 23, 25, 28, 29]. If extrapolated mortality risks for the trial control arm are higher than those observed in historical population data, the fitting should be considered problematic, as patients in the control arm can switch to pembrolizumab or other new and efficacious therapies that became available in recent years. Additionally, patients enrolled in the KEYNOTE NSCLC trials were relatively healthier than the general metastatic NSCLC population tracked by SEER because of stringent trial inclusion/exclusion criteria [32-36]. Thus, having higher extrapolated mortality risks for the chemotherapy arm than in historical patients likely underestimates the 
arm's longer-term survival. Indeed, Insinga et al. found that the longer-term survival of the pembrolizumab arm and the absolute magnitude of benefit of the pembrolizumab regimen were underestimated in this situation [22].

All the trial KMs incorporated survival effects of subsequent treatment, and thus the extrapolated survival curves reflected survival effects contributed by subsequent treatment.

\subsubsection{Model Validation}

Vemer et al. [37] summarized five parts of validation in their Assessment of the Validation Status of Health-Economic decision models (AdViSHE) tool, including validation of the conceptual model, model inputs, the computerized model, model outcomes, and others. Among the 14 publications, only Huang et al. (KEYNOTE-024) and Loong et al. described all five parts of validation performed by expert opinions and comparing with real-world data (RWD) in the AdViSHE tool [24, 29]. She et al., Weng et al. and Zhou et al. did not report validation in their papers $[18,19,25]$. The rest of the papers mainly focused on discussing validation of the long-term survival extrapolation or transition probability estimation, i.e., validation of model outcomes [16, 20-23, 26, 28, 30, 31].

Per suggestions from Vemer et al. validation of model outcomes can be done through face validity testing, cross validation testing, validation using alternative input data, and validation against empirical data [37]. Four studies used the face validation technique such as visual inspection and expert opinions to validate survival extrapolation results [20, $24,28,29]$. Four studies cross-validated survival extrapolation results with data from a different clinical trial or RWD $[24,26,28,29]$. Among these four studies, three only crossvalidated survival of chemotherapy-treated patients due to lack of long-term clinical trial data or long-term RWD on survival of patients treated with pembrolizumab regimens at the time when these studies were conducted [24, 26, 29]. Chouaid et al. cross-validated survival of patients treated with pembrolizumab monotherapy using published results of KEYNOTE-001, a single-arm study examining pembrolizumab's treatment effects on patients with advanced NSCLC with a median follow-up of 10.9 months [6, 28]. Six studies constructed long-term OS of chemotherapy-treated patients based on SEER [20-22, 24, 26, 31]. Three studies crossvalidated model-estimated survival results with the original trial data $[16,23,30]$.

\subsection{Cost Calculation Methods}

Table 3 summarizes the cost calculation methods used in the reviewed studies.

\subsubsection{Cost Categories}

All 14 studies limited cost calculations to direct medical costs, reflective of study perspectives. Major cost categories (drug acquisition/administration, disease management, adverse event $[\mathrm{AE}]$ costs) were included in all studies. Terminal care costs were captured by most studies except Liao et al., She et al. and Zhou et al [18, 19, 30]. Chouaid et al. also included transportation costs, categorizing them as direct medical costs [28].

Several KEYNOTE NSCLC trials were limited to patients whose tumors expressed PD-L1. For example, KEYNOTE-010 and KEYNOTE-042 enrolled patients with a TPS of $\geq 1 \%$, whereas KEYNOTE-024 enrolled patients with a TPS of $\geq 50 \%$. Most CEAs based on these trials included PD-L1 testing costs. Huang et al. (KEYNOTE-024) and Huang et al. (KEYNOTE-042) conducted a comparison of pembrolizumab versus chemotherapy assuming PD-L1 testing was performed as routine practice and thus did not include PD-L1 test costs in the base case. Both, however, added PD-L1 test costs in scenario analyses and found it had little impact on ICERs [24, 26].

Consistent with real-world clinical practice, patients could switch to subsequent therapies after treatment discontinuation in all KEYNOTE NSCLC trials. Therefore, postdiscontinuation treatment costs should be included to accurately reflect real-world practice and costs. Thirteen studies included subsequent treatment costs, most of which reported estimated ICERs were sensitive to post-discontinuation costs $[18,21,22,24-26,29]$. Liao et al. did not include postdiscontinuation treatment costs [30].

\subsubsection{Treatment Durations}

Two treatment duration approaches, trial-based time on treatment (ToT) and treat to progression (TTP), were applied in most studies. Five studies used trial-based ToT capped with maximum treatment durations per trial protocols and FDA recommendation (e.g., 35 cycles for pembrolizumab) to measure treatment duration [20-22, 24, 26]. Four studies applied the TTP approach, with Chouaid et al. and Weng et al. also incorporating maximum treatment durations $[25$, 28-30]. Georgieva et al. modeled treatment to end either upon progression or TRAEs [16]. The remaining studies reported applying a maximum treatment duration without specifying further details $[18,19,23,31]$. 


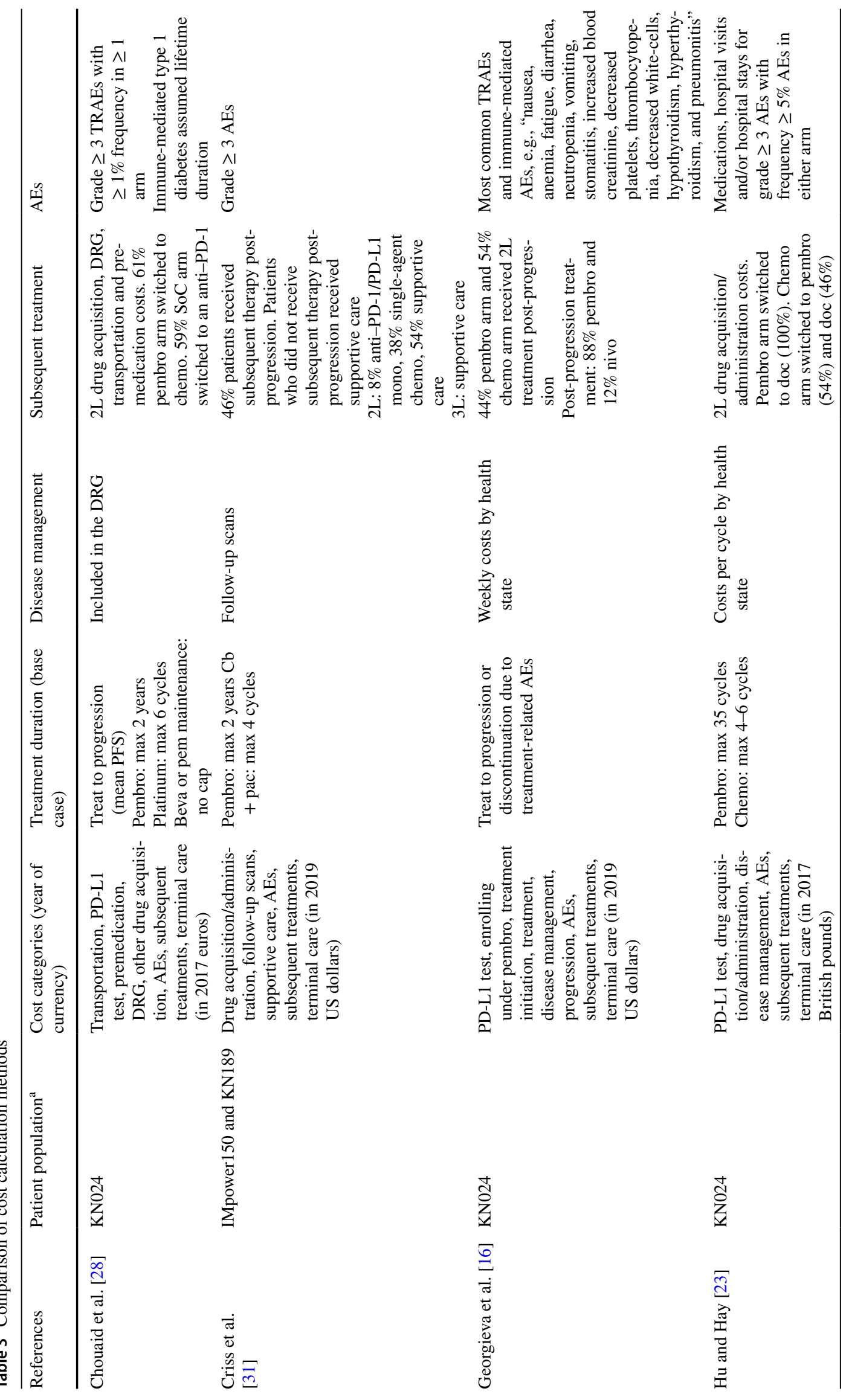




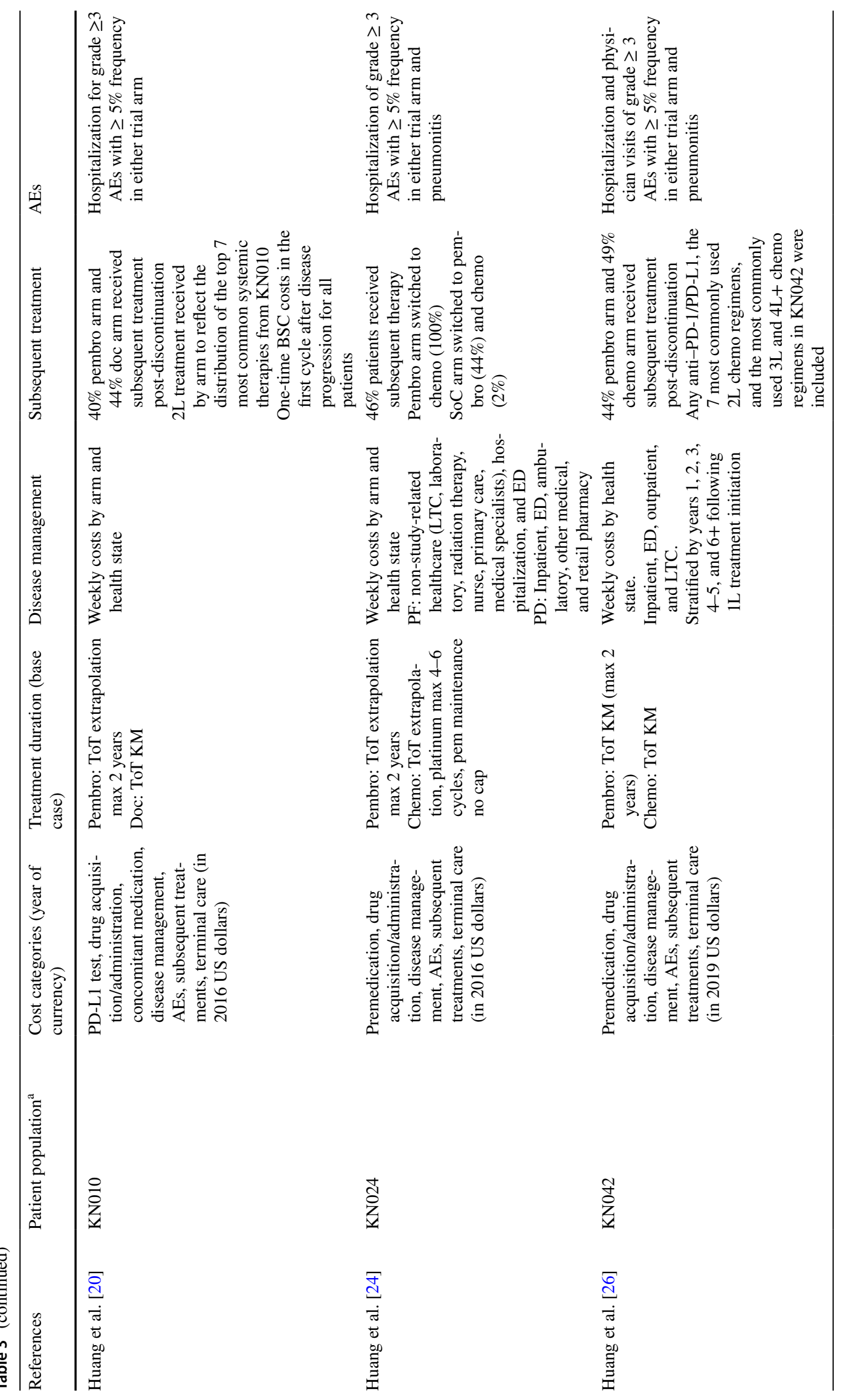




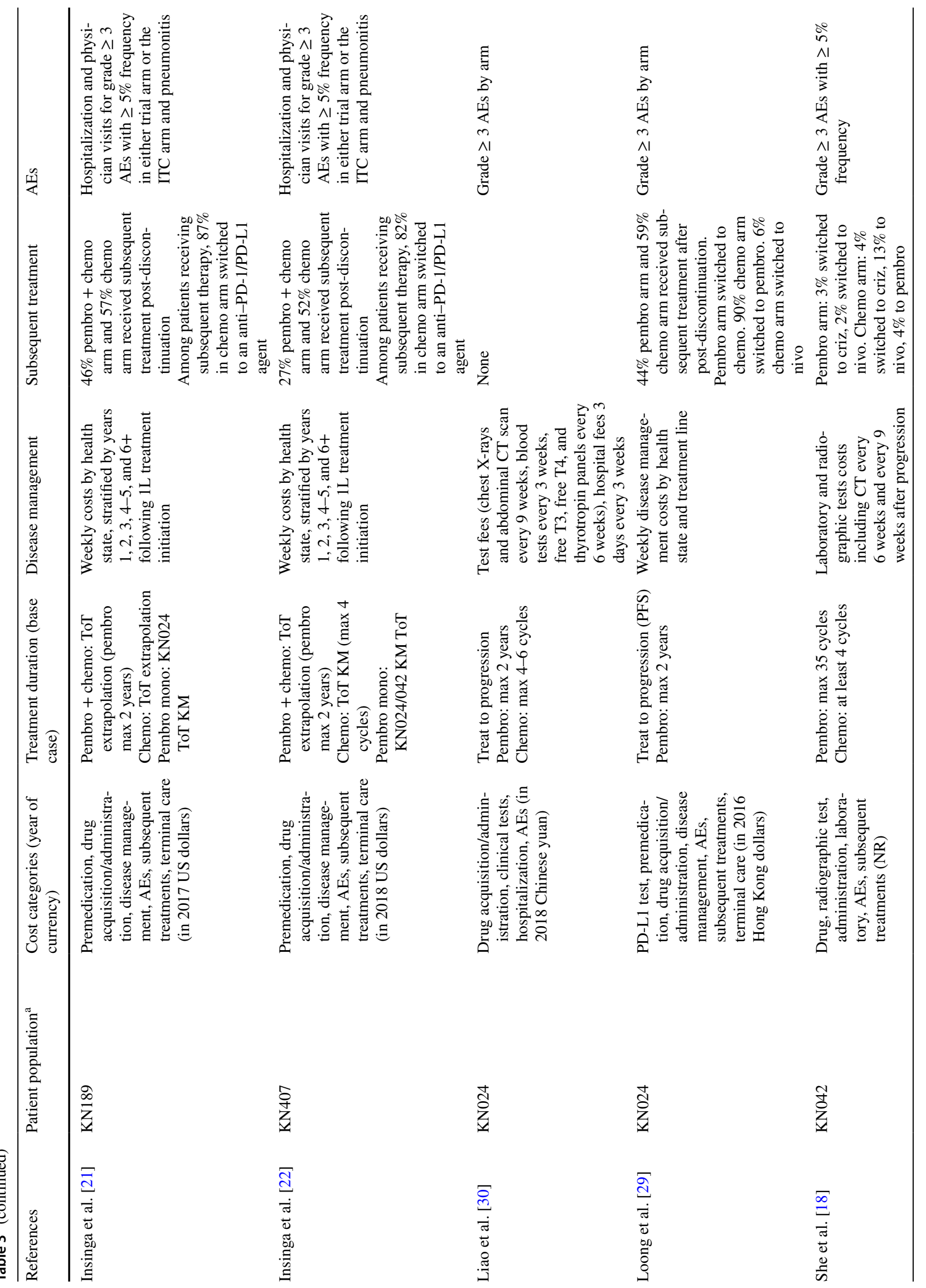




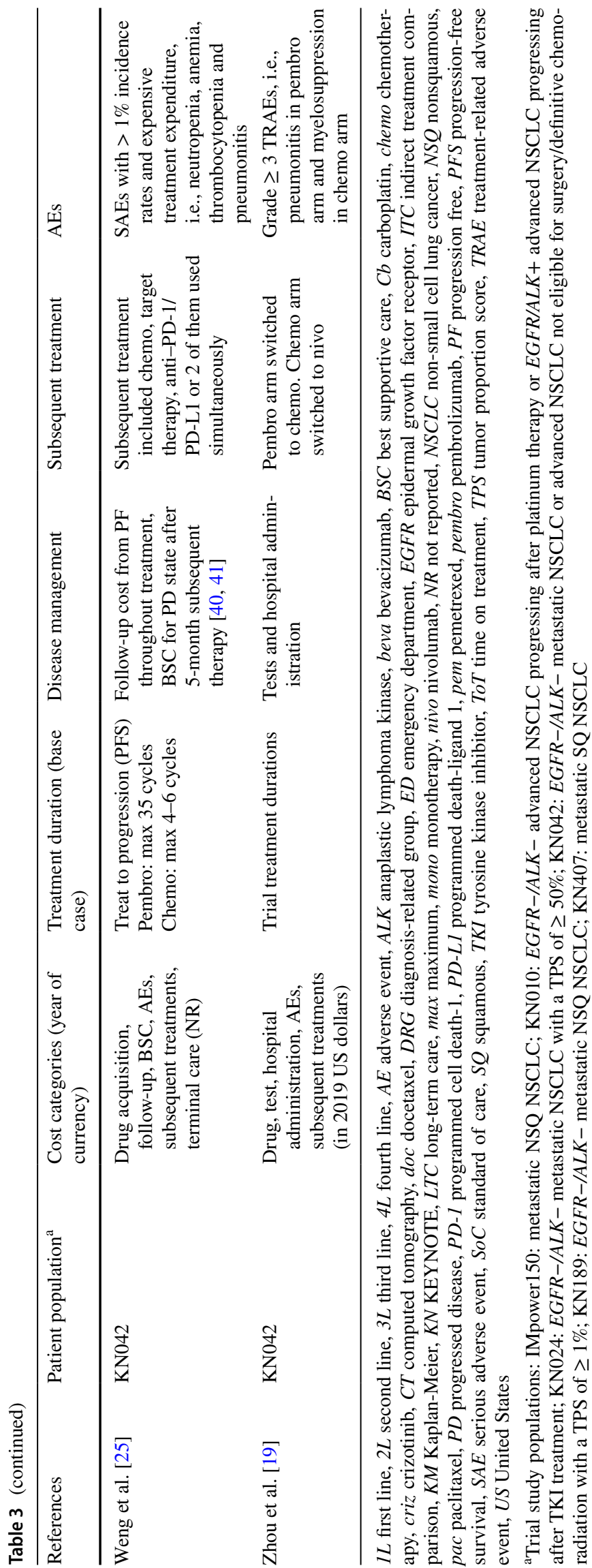

TTP does not necessarily produce estimates corresponding to actual patient treatment durations. In all pembrolizumab NSCLC clinical trials, as in real-world clinical practice, patients can discontinue treatment before disease progression due to safety, intercurrent illness, protocol noncompliance, or investigator/patient preference. Patients can also continue treatment after disease progression if the investigator considers patients can continue benefiting from pembrolizumab [20-22, 24, 26]. TTP approaches can overestimate treatment costs relative to actual observed ToT. For example, in the pembrolizumab plus chemotherapy arm in KEYNOTE-189, Gandhi et al. reported median PFS of 8.8 months versus median duration of treatment for pembrolizumab of 6.9 months, as estimated based on the median number of administrations [32]. Huang et al. (KEYNOTE-010) reported that when applying TTP without the 2-year cap to pembrolizumab-treated patients, the estimated ICER increased from $\$ 169 \mathrm{~K} / \mathrm{QALY}$ in the base case using trial-based ToT to \$215K/QALY. Applying TTP with the 2-year cap reduced the ICER to \$167K/QALY [20].

\subsubsection{Disease Management Costs}

Non-drug disease management costs were incorporated across the 14 studies at different levels of detail. For example, Huang et al. (KEYNOTE-024) included costs of longterm care, laboratory tests, radiation therapy, nurse/primary/ specialist care, hospitalization, and emergency department (ED) use for PF state disease management, and costs of hospitalization, ED use, ambulatory care, other medical services, and retail pharmacy for PD state disease management [24]. In contrast, Criss et al., Liao et al. and She et al. only counted radiographic and/or laboratory test fees as disease management costs [18, 30, 31].

As disease management in PD tends to be more costly than in PF, disease management costs were applied by health state in nine studies. Three studies further stratified healthstate-based management costs by years after treatment initiation. Based on cost inputs reported in these studies, disease management costs declined sharply over the first 6 years after treatment initiation $[21,22,26]$. Therefore, models that use a fixed disease management cost based on short-term follow-up data after treatment initiation may substantially overestimate costs compared with models that allow costs to decline over time based on extended follow-up data. As a result, total costs for treatments that extend survival time, such as pembrolizumab regimens, will be overestimated and thus undervalued if a single fixed disease management cost is applied. 


\subsubsection{Subsequent Treatment Costs}

Not all patients who discontinue treatment receive subsequent treatment. Patients with clinical progression/deterioration may not be candidates for another line of therapy, while others may opt for no further treatment. The proportions of post-discontinuation patients who received subsequent treatment in the pembrolizumab versus chemotherapy arms were $40 \%$ and $44 \%$ in KEYNOTE-010 [20], 44\% and 59\% in KEYNOTE-024 [29], 44\% and 49\% in KEYNOTE-042 [26], $46 \%$ and $57 \%$ in KEYNOTE- 189 [21], and $27 \%$ and $52 \%$ in KEYNOTE-407, respectively [22]. Five studies accounted for these values as reported [20-22, 26, 29]. Three studies included proportions of discontinued patients receiving subsequent treatment implicitly in proportions of patients receiving subsequent treatment [18, 24, 28]. Criss et al. and Georgieva et al. used proportions of progressed patients receiving subsequent treatment as a proxy for proportions of discontinued patients receiving subsequent treatment [16, 31].

Among studies including subsequent treatment costs, ten applied different post-discontinuation regimens across treatment arms and three assumed both arms switched to the same post-discontinuation regimens and had the same patient distribution across regimens, including anti-PD-1/ PD-L1 medications [16, 25, 31]. This assumption contradicts actual trial observations, i.e., subsequent anti-PD-1/ PD-L1 medication use was substantially higher in chemotherapy arms. Therefore, incorporation of equivalent use in the pembrolizumab regimen arms without adjustments to efficacy biases cost-effectiveness results against pembrolizumab regimens [32, 35].

\subsubsection{Adverse Event Costs}

Twelve studies included grade $\geq 3$ AEs [18-24, 26, 28-31], and three studies only considered TRAEs and/or immunemediated AEs [16, 19, 28]. Seven and two studies included AEs with $\geq 5 \%[18,20-24,26]$ and $\geq 1 \%$ frequency [25, 28 ], respectively. One study counted AE-related hospitalization costs only [20].

Ideally, all AEs regardless of causality, grade, severity, and frequency of observation should be included in a CEA in order to fully capture AE costs in each arm. However, some AEs observed from KEYNOTE NSCLC trials belong to one or more of the following categories: not caused by treatment, low-grade, less severe, and low-frequency [32-36]. For model simplification purposes, these AEs can be excluded.

\subsection{Utility Analysis Methods}

Table 4 presents the utility analysis methods used in the 14 studies.

\subsubsection{Data Sources}

Two data sources provided utility values to these studies: clinical trial data and published literature. Most pembrolizumab NSCLC trials (KEYNOTE-010, KEYNOTE-024, KEYNOTE-189, and KEYNOTE-407) collected utility data from patients using instruments such as the EuroQol 5-dimension, 3-level (EQ-5D 3L) questionnaire or the European Organization for the Research and Treatment of Cancer Quality of Life Questionnaire. Six studies based on these trials applied utility values calculated from trial-collected EQ-5D 3L survey data [20-22, 24, 28, 29]. The other four elicited utility values from published literature $[16,23,30$, 31]. The literature cited the most was a UK-based publication authored by Nafees et al. [42], which evaluated utility values for health-state vignettes where patients with NSCLC were treated with second-line chemotherapies based on a survey conducted in a convenience sample of the general UK population. Thus, there are limitations associated with applying these data to pembrolizumab patients, reflecting the time period of the study (before pembrolizumab availability and other potential changes to clinical practice), a lack of geographically representative data for establishing health-state values, limited applicability of second-line data, and a lack of direct health-related quality of life measurement in pembrolizumab-treated patients with NSCLC and the relevant trial comparator.

Quality of life data were not collected in the KEYNOTE-042 trial. Studies based on this trial used utility data collected from KEYNOTE-024 [26], published literature $[19,25]$, or a combination [18]. KEYNOTE-042 is a subsequent trial to KEYNOTE-024, expanding the patient population from TPS $\geq 50 \%$ to TPS $\geq 1 \%$. It is unknown whether KEYNOTE-042 patients with TPS of 1-49\% share the same utility values with KEYNOTE-024 patients, though a previous analysis did not observe substantive differences in utilities for a given health state by PD-L1 expression status [43].

\subsubsection{Analysis Approaches}

Two approaches were used to incorporate utilities in the cost-effectiveness models: progression status and time to death (TTD). The former assumes utility values for patients vary by NSCLC disease progression status, whereas the latter assumes utility values are affected by the patient's proximity to death and measures utility values accordingly. TTD potentially allows more detailed utility measurement compared with the progression status approach, which only distinguishes utility values before and after progression. According to Hatswell et al. patients with advanced or metastatic melanoma will exhibit a rapid decrease in utility in the last 180 days before death and using the progression status 
Table 4 Comparison of utility analysis methods

\begin{tabular}{|c|c|c|c|c|}
\hline References & Population $^{\mathrm{a}}$ & Data source & Analysis approach (base case) & Utility values (base case) $^{\mathrm{b}}$ \\
\hline Chouaid et al. [28] & KN024 & KN024 French values & Progression status & $\begin{array}{l}\text { PF without AE: } 0.778 \\
\text { PF with AE: } 0.687 \\
\text { PD: } 0.641\end{array}$ \\
\hline Criss et al. [31] & $\begin{array}{l}\text { IMpower150 and } \\
\text { KN189 }\end{array}$ & Insinga et al. [21] & TTD & $\begin{array}{l}\geq 360 \text { days: } 0.834 \\
(180,360): 0.765 \\
(30,180): 0.709 \\
<30: 0.563\end{array}$ \\
\hline Georgieva et al. [16] & KN024 & Nafees et al. [42] & $\begin{array}{l}\text { Progression status } \pm \text { NICE EoL approach } \\
\quad[47]\end{array}$ & $\begin{array}{l}\text { PF: uniform }(0.563,0.743) \\
\text { PD: uniform }(0.383,0.563)\end{array}$ \\
\hline Hu and Hay [23] & KN024 & $\begin{array}{l}\text { Brown et al. [48], Chouaid } \\
\text { et al. [49], and Nafees et al. } \\
\text { [42] }\end{array}$ & Progression status by treatment line/arm & $\begin{array}{l}\text { PF: } \\
\text { 1L pembro } 0.71 \text {; chemo } 0.68 \\
\text { 2L pembro } 0.67 \text {; chemo } 0.65 \\
\text { PD: } \\
\text { 1L pembro } 0.67 \text {; chemo } 0.67 \\
\text { 2L pembro } 0.59 \text {; chemo } 0.59 \\
\text { AE disutility applied }\end{array}$ \\
\hline Huang et al. [20] & KN010 & KN010 global values & TTD & $\begin{array}{l}\geq 360: 0.807 \\
(180,360): 0.728 \\
(90,180): 0.688 \\
(30,90): 0.602 \\
<30: 0.396 \\
\text { Grade } \geq 3 \text { AE disutility applied }\end{array}$ \\
\hline Huang et al. [24] & KN024 & KN024 global values & TTD & $\begin{array}{l}\geq 360: 0.805 \\
(180,360): 0.726 \\
(30,180): 0.632 \\
<30: 0.537\end{array}$ \\
\hline Huang et al. [26] & KN042 & KN024 & TTD & $\begin{array}{l}\geq 360: 0.808 \\
(180,360): 0.706 \\
(30,180): 0.625 \\
<30: 0.555\end{array}$ \\
\hline Insinga et al. [21] & KN189 & KN189 US values & TTD & $\begin{array}{l}\geq 360: 0.834 \\
(180,360): 0.765 \\
(30,180): 0.709 \\
<30: 0.563\end{array}$ \\
\hline Insinga et al. [22] & KN407 & KN407 US values & TTD & $\begin{array}{l}\geq 360: 0.842 \\
(180,360): 0.814 \\
(30,180): 0.737 \\
<30: 0.568\end{array}$ \\
\hline Liao et al. [30] & KN024 & Nafees et al. [42] & Progression status & $\begin{array}{l}\text { PF: } 0.65 \\
\text { PD: } 0.47\end{array}$ \\
\hline Loong et al. [29] & KN024 & KN024 global values & TTD & $\begin{array}{l}\geq 360: 0.805 \\
(180,360): 0.726 \\
(30,180): 0.632 \\
<30: 0.537\end{array}$ \\
\hline She et al. [18] & KN042 & $\begin{array}{l}\text { Derived from Nafees et al. } \\
\text { [42] and KN024 QLQ-C30 } \\
\text { QOL scores [50] }\end{array}$ & Progression status by treatment arm & $\begin{array}{l}\text { PF: pembro } 0.691 \text {; chemo } 0.653 \\
\text { PD: } 0.473\end{array}$ \\
\hline Weng et al. [25] & KN042 & Hu and Hay [23] & Progression status by treatment arm & $\begin{array}{l}\text { PF: pembro } 0.71 \text {; chemo } 0.68 \\
\text { PD: pembro } 0.67 \text {; chemo } 0.67\end{array}$ \\
\hline Zhou et al. [19] & KN042 & Huang et al. [20] & Progression status & $\begin{array}{l}\text { PF: } 0.761 \\
\text { PD: } 0.687\end{array}$ \\
\hline
\end{tabular}

$1 L$ first line, $2 L$ second line, $A E$ adverse event, $A L K$ anaplastic lymphoma kinase, chemo chemotherapy, EGFR epidermal growth factor receptor, EoL end-of-life, KN KEYNOTE, NICE National Institute for Health and Care Excellence, NSCLC non-small cell lung cancer, NSQ nonsquamous, $P D$ progressed disease, pembro pembrolizumab, $P F$ progression free, $Q L Q$-C30 Quality of Life Questionnaire-Core 30-item module, $Q O L$ quality of life, $S Q$ squamous, $T K I$ tyrosine kinase inhibitor, TPS tumor proportion score, TTD time to death, US United States

${ }^{a}$ Trial study populations: IMpower150: metastatic NSQ NSCLC; KN010: EGFR-/ALK- advanced NSCLC progressing after platinum therapy or $E G F R / A L K+$ advanced NSCLC progressing after TKI treatment; KN024: $E G F R-/ A L K-$ metastatic NSCLC with a TPS of $\geq 50 \%$; KN042: $E G F R-/ A L K-$ metastatic NSCLC or advanced NSCLC not eligible for surgery/definitive chemoradiation with a TPS of $\geq 1 \%$; KN189: EGFR-/ $A L K-$ metastatic NSQ NSCLC; KN407: metastatic SQ NSCLC.

${ }^{\mathrm{b}} \mathrm{TTD}$ values correspond to days until death 
approach cannot capture contributions to the change caused by other factors [44].

Georgieva et al. [16] cited the end-of-life framework suggested by the British National Institute of Clinical Excellence (NICE) and assigned a utility value of 1.0 to patients whose OS was extended for $>3$ months by pembrolizumab versus chemotherapy.

Three studies applied AE disutility explicitly in modeling $[20,23,28]$. Two studies did not include AE disutility explicitly or implicitly in modeling $[19,25]$. The rest of the studies included AE disutility implicitly within the progression status or TTD utility values $[16,18,21,22,24,26$, 29-31]. None of the studies compared QALYs and ICERs with and without taking AE disutility into consideration, and thus we could not conclude whether excluding AE disutility would impose a significant impact on the cost-effectiveness estimation.

\subsubsection{Utility Values}

The utility value inputs used in the 14 studies varied by indication, data source, and analysis approach. Nafees et al. estimated utility values of the three NSCLC health states (responding, stable, and progressive) to be $0.673,0.653$, and 0.473 , respectively [42]. These values were lower than estimates for previously treated patients with NSCLC in KEYNOTE-010 reported by Huang et al. which were 0.761 for PF and 0.687 for PD [20]. The discrepancy can be attributed to three factors. First, with the introduction of new health technologies, utility values can increase over time. For example, in a meta-analysis of chronic kidney disease patients' utilities, utility values of transplant patients increased from 0.66 to 0.85 from the 1980 s to the 2000s [45]. Utility values estimated by Nafees et al. do not reflect the development of less toxic, more efficacious therapeutic options for NSCLC in the 10-15 years since that study was conducted. The second factor refers to whether utilities are elicited from patients or the general public. For example, the general public may be more likely to overestimate NSCLC's impact on patients' quality of life than cancer patients [46]. Finally, the discrepancy may be partially attributed to different instruments used to measure quality of life in the two studies.

Between the two approaches, patients with $\geq 360$ days to death in the TTD approach had higher utility values compared to the PF utility value with the progression status approach, while patients approaching death had lower utility values than the PD utility value. For example, Huang et al. used KEYNOTE-010 trial data to calculate utility inputs for both progression status and TTD approaches. The utility values related to PF and PD were estimated to be 0.761 and 0.687 , while the utility values for patients with $\geq 360,180-360,90-180,30-90$, and $<30$ days to death were estimated to be $0.807,0.728,0.688,0.602$, and 0.396 , respectively [20], suggesting that the progression status approach underestimates patients' quality of life when they have longer remaining life and overestimates patients' quality of life when they approach death in KEYNOTE-010. This finding may indicate that TTD is a more appropriate approach to estimate utility values of advanced NSCLC patients, while we acknowledge that this conclusion may not be generalized to other types of cancer, especially those with high survival rates.

\section{Discussion}

This is the first comprehensive review of assumptions and methodologies applied in published CEA literature comparing pembrolizumab regimens with chemotherapy/immunotherapy in treating advanced NSCLC. Other existing CEA literature review publications including pembrolizumab focused on ranking the cost-effectiveness among different treatments by directly comparing ICERs, without comparing and discussing the appropriateness of the applied methodologies $[9,10]$.

Included publications in our review had varying levels of reporting quality. Important methodological or input information was missing or not clearly specified in several studies, making comparison difficult. For example, Criss et al. did not report the time horizon of the CEA [31]. Chouaid et al. and Zhou et al. did not indicate whether or how nondrug disease management costs were applied in their models $[19,28]$. In Liao et al. many details of the modeling approach and cost inputs were not specified [30]. Loong et al. reported both TTP and extrapolated ToT as the treatment duration measurement approach, though PFS was actually used (based on communication with a study author) [29]. Furthermore, several studies used ambiguous terms. For example, Georgieva et al. reported including pembrolizumab-arm patient enrollment costs without explaining what these costs were [16]. She et al. and Zhou et al. included "administration" and "hospital administration" costs, without clarifying whether these costs represented drug administration costs or hospital administrative costs [18, 19]. To evaluate the quality of and to interpret the results of CEA publications, it will be critical for authors to follow current CEA reporting guidelines by reporting key elements of modeling methods and inputs, using clear and specific terminology [51].

Through summarizing and comparing methodologies applied in different studies and cost-effectiveness results, we found the choice of methodologies can lead to important differences in research findings and sometimes alter study conclusions.

Some studies employed approaches based on strong assumptions for model simplification purposes or due to 
data limitations. For example, assuming time independency of survival probabilities in a Markov model, TTP in estimating treatment costs, the same management costs for PF and PD states, or the same post-discontinuation regimens across arms are all contradicted by empirical experience from clinical trials and/or population data. The direction of the bias sometimes can be predicted. For example, neglecting postdiscontinuation treatment costs or assuming the same cost in each arm will underestimate pembrolizumab's cost offsets due to the use of more extensive and expensive secondline regimens following chemotherapy treatment discontinuation, and therefore biases the results towards favoring chemotherapy. In contrast, assigning a perfect utility score to pembrolizumab-treated patients with $>3$ months life extension versus chemotherapy can influence the ICER in a direction favoring pembrolizumab. Finally, when a questionable approach affects both arms or when multiple inappropriate approaches confound results in opposite directions, it can be impossible to predict the direction of bias. Researchers should acknowledge and report the potential bias introduced through such assumptions and readers should be very cautious when interpreting results.

As many markets require demonstration of cost-effectiveness for new drugs, studies with opposite conclusions around pembrolizumab's cost-effectiveness in treating the same indication for the same markets can lead to confusion. For example, Georgieva et al. reported ICERs of $£ 45 \mathrm{~K} /$ QALY [16] in comparison with $£ 87 \mathrm{~K} / \mathrm{QALY}$ reported by $\mathrm{Hu}$ and Hay [23] for the KEYNOTE-024 indication from the UK National Health Service perspective. The differences in estimated ICERs can be attributed to many factors, such as assigning a perfect utility value to pembrolizumab-treated patients with $>3$ months life extension compared with the chemotherapy arm in Georgieva et al. [16] and using the exponential extrapolation as the base for transition probability estimation in $\mathrm{Hu}$ and Hay [23]. Results from the two studies ended up on opposite sides of the £50K/QALY willingness-to-pay threshold for end-of-life therapies recommended by NICE [52], which theoretically could have led to divergent coverage/reimbursement decisions affecting patients' access to pembrolizumab.

\subsection{Limitations}

Several limitations are acknowledged for this literature review. First, the study focused on peer-reviewed CEA publications. We acknowledge that Health Technology Assessment (HTA) appraisals may include insightful discussions on limitations of research design and/or data inputs used in selected CEAs. However, we did not find any HTA appraisal published on pembrolizumab as a treatment for NSCLC when the search was conducted. Second, only studies published in the English language were included. Despite these studies covering multiple geographic regions, it should be noted that they may not fully represent pembrolizumab CEA publications in other languages. Third, no quality assessment was performed on searched studies as part of the inclusion criteria. All CEAs of pembrolizumab regimens as treatment for NSCLC regardless of quality were summarized and compared for the purpose of critically evaluating and comparing applied methodologies. Fourth, some studies missed reporting or failed to report in detail important methodological aspects, and these elements could not be compared with other studies. Finally, due to the number of studies and limitations in information reported, we could not precisely identify and quantify all sources of variation in ICERs across studies, though some relevant factors and their perceived impact have been identified herein.

\section{Conclusion}

With growing healthcare expenditures worldwide, more and more healthcare payers rely on CEAs to make drug coverage and reimbursement decisions. These decisions can affect clinical guidelines and practice and impact patients' access to drugs. In such circumstances, it is important for the CEAs used for decision making to meet high standards and produce unbiased results. In this literature review, we found the quality of published CEAs varies greatly, and that questionable CEA methodologies could significantly bias results and alter study conclusions. This illustrates the importance of payers, policy makers, and the scientific community to carefully examine study designs and assumptions when using CEAs for evidence-based decision-making.

Acknowledgements The research was supported by Merck Sharp \& Dohme Corp., a subsidiary of Merck \& Co., Inc., Kenilworth, NJ, USA. The authors thank Dr. Thomas Burke (Merck \& Co., Inc., Kenilworth, NJ, USA) for his contribution to the research design and manuscript revision. The authors also thank Dr. Ina Nikolaeva, and Ms. Sheila Erespe (Merck \& Co., Inc., Kenilworth, NJ, USA) for her copyediting and formatting assistance.

\section{Declarations}

Conflict of interest Nan Qiao and Ralph Insinga are employees of Merck \& Co., Inc., Kenilworth, NJ, USA. Gilberto de Lima Lopes Junior is an employee of the University of Miami Clinical Oncology Sector, which has received a research grant from Merck \& Co., Inc., Kenilworth, NJ, USA. John Cook and Martin Sénécal are employees of Complete HEOR Solutions, which received funding from Merck \& Co., Inc., Kenilworth, NJ, USA.

Author contributions NQ developed the study concept, designed and planned the study, searched and collected the reviewed literature, analyzed the data, interpreted the results, and drafted the manuscript. RI developed the study concept, designed and planned the study, and inter- 
preted the results. GdLL Jr planned the study. JC and MS interpreted the results. All authors critically reviewed and/or revised the manuscript, approved the content of the manuscript and its submission, and agreed to be accountable for all aspects of the manuscript.

Open Access This article is licensed under a Creative Commons Attribution-NonCommercial 4.0 International License, which permits any non-commercial use, sharing, adaptation, distribution and reproduction in any medium or format, as long as you give appropriate credit to the original author(s) and the source, provide a link to the Creative Commons licence, and indicate if changes were made. The images or other third party material in this article are included in the article's Creative Commons licence, unless indicated otherwise in a credit line to the material. If material is not included in the article's Creative Commons licence and your intended use is not permitted by statutory regulation or exceeds the permitted use, you will need to obtain permission directly from the copyright holder. To view a copy of this licence, visit http://creativecommons.org/licenses/by-nc/4.0/.

\section{References}

1. Bray F, Ferlay J, Soerjomataram I, Siegel RL, Torre LA, Jemal A. Global cancer statistics 2018: GLOBOCAN estimates of incidence and mortality worldwide for 36 cancers in 185 countries. CA Cancer J Clin. 2018;68:394-424.

2. Non-Small Cell Lung Cancer. American Cancer Society. https ://www.cancer.org/cancer/non-small-cell-lung-cancer.html. Accessed 2 May 2020.

3. Molina JR, Yang P, Cassivi SD, Schild SE, Adjei AA. Non-small cell lung cancer: epidemiology, risk factors, treatment, and survivorship. Mayo Clin Proc. 2008;83:584-94.

4. Zappa C, Mousa SA. Non-small cell lung cancer: current treatment and future advances. Transl Lung Cancer Res. 2016;5:288-300.

5. Doroshow DB, Sanmamed MF, Hastings K, Politi K, Rimm DL, Chen L, Melero I, Schalper KA, Herbst RS. Immunotherapy in non-small cell lung cancer: facts and hopes. Clin Cancer Res. 2019;25:4592-602.

6. Garon EB, Rizvi NA, Hui R, Leighl N, Balmanoukian AS, Eder JP, Patnaik A, Aggarwal C, Gubens M, Horn L, et al. Pembrolizumab for the treatment of non-small-cell lung cancer. N Engl J Med. 2015;372:2018-28.

7. Hematology/Oncology (Cancer) Approvals and Safety Notifications. http://wayback.archive-it.org/7993/20170111064250/http:// www.fda.gov/Drugs/InformationOnDrugs/ApprovedDrugs/ucm27 9174.htm. Accessed 21 Sept 2019.

8. Drugs@FDA: FDA Approved Drug Products. https://www.acces sdata.fda.gov/scripts/cder/daf/index.cfm?event $=$ BasicSearc h.process. Accessed 18 Nov 2019.

9. da Veiga CRP, da Veiga CP, Drummond-Lage AP. Concern over cost of and access to cancer treatments: a meta-narrative review of nivolumab and pembrolizumab studies. Crit Rev Oncol Hematol. 2018;129:133-45.

10. Verma V, Sprave T, Haque W, Simone CB 2nd, Chang JY, Welsh JW, Thomas CR Jr. A systematic review of the cost and cost-effectiveness studies of immune checkpoint inhibitors. J Immunother Cancer. 2018;6:128.

11. Bravaccini S. What impacts the cost-effectiveness of PD-L1 testing in non-small cell lung cancer? Lung Cancer. 2019;132:152-3.

12. Norum J, Antonsen MA, Tollali T, Al-Shibli K, Andersen G, Svanqvist KH, Helbekkmo N. Pembrolizumab as second-line therapy in non-small cell lung cancer in northern Norway: budget impact and expected gain-a model-based analysis. ESMO Open. 2017;2:e00222.

13. Tartari F, Santoni M, Burattini L, Mazzanti P, Onofri A, Berardi R. Economic sustainability of anti-PD-1 agents nivolumab and pembrolizumab in cancer patients: recent insights and future challenges. Cancer Treat Rev. 2016;48:20-4.

14. Aguiar P Jr, Giglio AD, Perry LA, Penny-Dimri J, Babiker H, Tadokoro H, Lopes G Jr, De Mello RA. Cost-effectiveness and budget impact of lung cancer immunotherapy in South America: strategies to improve access. Immunotherapy. 2018;10:887-97.

15. Aguiar PN Jr, Perry LA, Penny-Dimri J, Babiker H, Tadokoro H, de Mello RA, Lopes GL Jr. The effect of PD-L1 testing on the cost-effectiveness and economic impact of immune checkpoint inhibitors for the second-line treatment of NSCLC. Ann Oncol. 2017;28:2256-63.

16. Georgieva M, da Lima JPSN, Aguiar P Jr, de Lopes GL Jr, Haaland B. Cost-effectiveness of pembrolizumab as first-line therapy for advanced non-small cell lung cancer. Lung Cancer. 2018;124:248-54

17. Garrison LP Jr, Pauly MV, Willke RJ, Neumann PJ. An overview of value, perspective, and decision context-a health economics approach: an ISPOR special task force report [2]. Value Health. 2018;21:124-30.

18. She L, Hu H, Liao M, Xia X, Shi Y, Yao L, Ding D, Zhu Y, Zeng $\mathrm{S}$, Shen L, et al. Cost-effectiveness analysis of pembrolizumab versus chemotherapy as first-line treatment in locally advanced or metastatic non-small cell lung cancer with PD-L1 tumor proportion score 1\% or greater. Lung Cancer. 2019;138:88-94.

19. Zhou K, Jiang C, Li Q. Cost-effectiveness analysis of pembrolizumab monotherapy and chemotherapy in the non-small-cell lung cancer with different PD-L1 tumor proportion scores. Lung Cancer. 2019;136:98-101.

20. Huang M, Lou Y, Pellissier J, Burke T, Liu FX, Xu R, Velcheti V. Cost-effectiveness of pembrolizumab versus docetaxel for the treatment of previously treated PD-L1 positive advanced NSCLC patients in the United States. J Med Econ. 2017;20:140-50.

21. Insinga RP, Vanness DJ, Feliciano JL, Vandormael K, Traore S, Burke T. Cost-effectiveness of pembrolizumab in combination with chemotherapy in the 1st line treatment of non-squamous NSCLC in the US. J Med Econ. 2018;21:1191-205.

22. Insinga RP, Vanness DJ, Feliciano JL, Vandormael K, Traore S, Ejzykowicz F, Burke T. Cost-effectiveness of pembrolizumab in combination with chemotherapy versus chemotherapy and pembrolizumab monotherapy in the first-line treatment of squamous non-small-cell lung cancer in the US. Curr Med Res Opin. 2019;35:1241-56.

23. $\mathrm{Hu} \mathrm{X}$, Hay JW. First-line pembrolizumab in PD-L1 positive nonsmall-cell lung cancer: a cost-effectiveness analysis from the UK health care perspective. Lung Cancer. 2018;123:166-71.

24. Huang M, Lou Y, Pellissier J, Burke T, Liu FX, Xu R, Velcheti V. Cost Effectiveness of pembrolizumab vs. standard-of-care chemotherapy as first-line treatment for metastatic NSCLC that expresses high levels of PD-L1 in the United States. Pharmacoeconomics. 2017;35:831-44.

25. Weng X, Luo S, Lin S, Zhong L, Li M, Xin R, Huang P, Xu X. Cost-utility analysis of pembrolizumab versus chemotherapy as first-line treatment for metastatic non-small cell lung cancer with different PD-L1 expression levels. Oncol Res. 2020;28(2):117-25.

26. Huang M, Lopes GL, Insinga RP, Burke T, Ejzykowicz F, Zhang Y, Feliciano JL. Cost-effectiveness of pembrolizumab versus chemotherapy as first-line treatment in PD-L1-positive advanced non-small-cell lung cancer in the USA. Immunotherapy. 2019;11:1463-78.

27. Briggs AH, Weinstein MC, Fenwick EA, Karnon J, Sculpher MJ, Paltiel AD. Model parameter estimation and uncertainty analysis: 
a report of the ISPOR-SMDM Modeling Good Research Practices Task Force Working Group-6. Med Decis Mak. 2012;32:722-32.

28. Chouaid C, Bensimon L, Clay E, Millier A, Levy-Bachelot L, Huang M, Levy P. Cost-effectiveness analysis of pembrolizumab versus standard-of-care chemotherapy for first-line treatment of PD-L1 positive ( $>50 \%$ ) metastatic squamous and nonsquamous non-small cell lung cancer in France. Lung Cancer. 2019;127:44-52.

29. Loong HH, Wong CKH, Leung LKS, Dhankhar P, Insinga RP, Chandwani S, Hsu DC, Lee MYK, Huang M, Pellissier J, et al. Cost effectiveness of PD-L1-based test-and-treat strategy with pembrolizumab as the first-line treatment for metastatic NSCLC in Hong Kong. Pharmacoecon Open. 2020;4:235-47.

30. Liao W, Huang J, Hutton D, Li Q. Cost-effectiveness analysis of first-line pembrolizumab treatment for PD-L1 positive, non-small cell lung cancer in China. J Med Econ. 2019;22:344-9.

31. Criss SD, Mooradian MJ, Watson TR, Gainor JF, Reynolds KL, Kong CY. Cost-effectiveness of atezolizumab combination therapy for first-line treatment of metastatic nonsquamous nonsmall cell lung cancer in the United States. JAMA Netw Open. 2019;2:e1911952.

32. Gandhi L, Rodriguez-Abreu D, Gadgeel S, Esteban E, Felip E, De Angelis F, Domine M, Clingan P, Hochmair MJ, Powell SF, et al. Pembrolizumab plus chemotherapy in metastatic non-small-cell lung cancer. N Engl J Med. 2018;378:2078-92.

33. Mok TSK, Wu YL, Kudaba I, Kowalski DM, Cho BC, Turna HZ, Castro G Jr, Srimuninnimit V, Laktionov KK, Bondarenko I, et al. Pembrolizumab versus chemotherapy for previously untreated, PD-L1-expressing, locally advanced or metastatic non-small-cell lung cancer (KEYNOTE-042): a randomised, open-label, controlled, phase 3 trial. Lancet. 2019;393:1819-30.

34. Reck M, Rodriguez-Abreu D, Robinson AG, Hui R, Csoszi T, Fulop A, Gottfried M, Peled N, Tafreshi A, Cuffe S, et al. Pembrolizumab versus chemotherapy for PD-L1-positive non-smallcell lung cancer. N Engl J Med. 2016;375:1823-33.

35. Paz-Ares L, Luft A, Vicente D, Tafreshi A, Gumus M, Mazieres J, Hermes B, Cay Senler F, Csoszi T, Fulop A, et al. Pembrolizumab plus chemotherapy for squamous non-small-cell lung cancer. N Engl J Med. 2018;379:2040-51.

36. Herbst RS, Baas P, Kim DW, Felip E, Perez-Gracia JL, Han JY, Molina J, Kim JH, Arvis CD, Ahn MJ, et al. Pembrolizumab versus docetaxel for previously treated, PD-L1-positive, advanced non-small-cell lung cancer (KEYNOTE-010): a randomised controlled trial. Lancet. 2016;387:1540-50.

37. Vemer P, Corro Ramos I, van Voorn GA, Al MJ, Feenstra TL. AdViSHE: a validation-assessment tool of health-economic models for decision makers and model users. Pharmacoeconomics. 2016;34:349-61

38. Beck JR, Kassirer JP, Pauker SG. A convenient approximation of life expectancy (the "DEALE"). I. Validation of the method. Am J Med. 1982;73:883-8.

39. Hoyle MW, Henley W. Improved curve fits to summary survival data: application to economic evaluation of health technologies. BMC Med Res Methodol. 2011;11:139.

40. Ellis PM, Leighl NB, Hirsh V, Reaume MN, Blais N, Wierzbicki R, Sadrolhefazi B, Gu Y, Liu D, Pilz K, Chu Q. A randomized, open-label phase II trial of volasertib as monotherapy and in combination with standard-dose pemetrexed compared with pemetrexed monotherapy in second-line treatment for non-small-cell lung cancer. Clin Lung Cancer. 2015;16:457-65.

41. Borghaei H, Paz-Ares L, Horn L, Spigel DR, Steins M, Ready NE, Chow LQ, Vokes EE, Felip E, Holgado E, et al. Nivolumab versus docetaxel in advanced nonsquamous non-small-cell lung cancer. N Engl J Med. 2015;373:1627-39.

42. Nafees B, Stafford M, Gavriel S, Bhalla S, Watkins J. Health state utilities for non small cell lung cancer. Health Qual Life Outcomes. 2008;6:84.

43. Huang M, Chandwani S, Insinga R, Burke T, Pellissier J, Pickard A. PCN345: health state utilities in metastatic NSCLC: a study of multiple immuno-oncology trials. Value Health. 2018;21:S72-3.

44. Hatswell AJ, Pennington B, Pericleous L, Rowen D, Lebmeier M, Lee D. Patient-reported utilities in advanced or metastatic melanoma, including analysis of utilities by time to death. Health Qual Life Outcomes. 2014;12:140.

45. Wyld M, Morton RL, Hayen A, Howard K, Webster AC. A systematic review and meta-analysis of utility-based quality of life in chronic kidney disease treatments. PLoS Med. 2012;9:e1001307-e1001307.

46. Insinga RP, Fryback DG. Understanding differences between selfratings and population ratings for health in the EuroQOL. Qual Life Res. 2003;12:611-9.

47. Stewart G, Eddowes L, Hamerslag L, Kusel J. HC2 - the impact of Nice's end-of-life threshold on patient access to new cancer therapies in England And Wales. Value Health. 2014;17:A6.

48. Brown J, Cook K, Adamski K, Lau J, Bargo D, Breen S, Chawla A. Utility values associated with advanced or metastatic non-small cell lung cancer: data needs for economic modeling. Expert Rev Pharmacoecon Outcomes Res. 2017;17:153-64.

49. Chouaid C, Agulnik J, Goker E, Herder GJ, Lester JF, Vansteenkiste J, Finnern HW, Lungershausen J, Eriksson J, Kim K, Mitchell PL. Health-related quality of life and utility in patients with advanced non-small-cell lung cancer: a prospective crosssectional patient survey in a real-world setting. J Thorac Oncol. 2013;8:997-1003.

50. Brahmer JR, Rodriguez-Abreu D, Robinson AG, Hui R, Csoszi T, Fulop A, Gottfried M, Peled N, Tafreshi A, Cuffe S, et al. Healthrelated quality-of-life results for pembrolizumab versus chemotherapy in advanced, PD-L1-positive NSCLC (KEYNOTE-024): a multicentre, international, randomised, open-label phase 3 trial. Lancet Oncol. 2017;18:1600-9.

51. Husereau D, Drummond M, Petrou S, Carswell C, Moher D, Greenberg D, Augustovski F, Briggs AH, Mauskopf J, Loder E. Consolidated Health Economic Evaluation Reporting Standards (CHEERS) — explanation and elaboration: a report of the ISPOR Health Economic Evaluation Publication Guidelines Good Reporting Practices Task Force. Value Health. 2013;16:231-50.

52. National Institute for Health and Care Excellence. Guide to the methods of technology appraisal 2013-process and methods. London: National Institute for Health and Care Excellence; 2013. 